\title{
Neural correlates of consciousness
}

\author{
BL Negrao, M Viljoen
}

Department of Physiology, School of Medicine, Faculty of Health Sciences, University of Pretoria, Pretoria, South Africa

\begin{abstract}
A basic understanding of consciousness and its neural correlates is of major importance for all clinicians, especially those involved with patients with altered states of consciousness. In this paper it is shown that consciousness is dependent on the brainstem and thalamus for arousal; that basic cognition is supported by recurrent electrical activity between the cortex and the thalamus at gamma band frequencies; and that some kind of working memory must, at least fleetingly, be present for awareness to occur. The problem of cognitive binding and the role of attention are briefly addressed and it is shown that consciousness depends on a multitude of subconscious processes. Although these processes do not represent consciousness, consciousness cannot exist without them
\end{abstract}

Keywords: Consciousness; Brainstem; Thalamocortical system; Memory

Received: 10-10-2008

Accepted: 18-12-2008

\section{Introduction}

Generally speaking, there are two opposing views about the extensiveness of neuronal involvement in consciousness. The holistic approach to consciousness emphasizes the perspective that all neurons in the brain collectively form the neural correlate(s) of consciousness, which therefore cannot be localized to a specific set of neural cells. ${ }^{1}$ In contrast, the neuronal specificity approach promotes the idea that consciousness depends on the formation of complex arrangements which can be pin-pointed to specific groups of neural cells. ${ }^{1}$ Under this approach, consciousness is believed to be a product of the processing of multiple brain regions, which in turn produce a high level of information integration. ${ }^{2}$ This paper touches on those neurobiological aspects that are reasonably well accepted to be necessary for consciousness, as well as the involvement of memory in consciousness.

\section{$40 \mathrm{~Hz}$ synchronized brain activity as a prerequisite for consciousness}

Consciousness entails desynchronized, low-amplitude brain activity ranging from 20 to $70 \mathrm{~Hz} \cdot{ }^{3}$ Embedded in this pattern of global desynchronization are phase-locked, synchronized

\section{Correspondence:}

Prof M Viljoen

Department of Physiology, School of Medicine, Faculty of Health

Sciences, University of Pretoria

Box 2043, Pretoria, 0001

email: mviljoen@medic.up.ac.za areas which oscillate steadily in unison at the gamma band frequency of $40 \mathrm{~Hz} .{ }^{4}$ These areas are believed to be coordinated by the intralaminar nuclei of the thalamus, however, it is the information flowing back and forth between the intralaminar thalamic nuclei and the cortex through the thalamocortical system which is believed to set up the $40 \mathrm{~Hz}$ oscillating loop. ${ }^{4}$ The neurons of the specific thalamic nuclei have been shown to activate specific cortical pyramidal cells and silence other brain areas via activation of $40 \mathrm{~Hz} \gamma$-aminobutyric acid (GABA) inhibitory interneurons. ${ }^{5}$ Consciousness is therefore believed to be possible only when the $40 \mathrm{~Hz}$ electrical hum is sustained among the brain circuits, with the recruitment of more cerebral networks allowing our consciousness to widen. ${ }^{4}$ Significant coupling in the gamma frequency has been shown to occur in both wakefulness and rapid-eye movement (REM) sleep, although the variability of gamma cycle duration is larger during sleep. ${ }^{6}$ Sensory perturbation is able to reset this gamma rhythm in awake but not sleep states $^{6}$, indicating that the cortex activates itself from within during sleep. ${ }^{4}$ During deep sleep, however, the intralaminar thalamic nuclei are inactive and no $40 \mathrm{~Hz}$ oscillations arise. ${ }^{4}$ Interestingly, gamma oscillations have been shown to emerge about 150-300 msec after the onset of the stimulus, which is believed to be more or less when the stimuli acquire meaning. ${ }^{6}$ An increase in gamma power has been shown to be associated with an increase in the working memory load during the period of retention, specifically above the prefrontal cortex. ${ }^{6}$ Moreover, gamma oscillations 
are believed to play a central role in the timing mechanism essential for synaptic plasticity, since strengthening of a synapse occurs if a pre-synaptic input is followed by a burst discharge in the post-synaptic neuron within $40 \mathrm{msec}^{6}$

\section{The thalamocortical system and brain stem reticular formation participation in consciousness}

Due to their importance for arousal, one would instinctively expect the brain stem ascending reticular activating system (ARAS) and the thalamocortical system to be essential for consciousness. Based on the integration of information from a multitude of studies, clarity about the role of these systems in consciousness is slowly emerging. Zeman believes that consciousness relies on a fast integration of information which is made possible by thalamocortical re-entrant loops. ${ }^{7}$ According to Llinás ${ }^{8}$, consciousness is generated in the closed-loop system of the cortex, thalamus and reticular formation by neurons that fire spontaneously. Massimini believes that consciousness depends on the ability of the brain to integrate information, which itself depends on effective connectivity among functionally specialized regions of the thalamocortical system. ${ }^{9}$ This system is deemed to be turned on and off by brainstem neuromodulation3 with the brainstem believed to activate the cortex via the ARAS. ${ }^{10}$ It is believed that brainstem nuclei can modulate the rhythmicity between the thalamus and cortex and may therefore affect the rhythmic bursting of thalamocortical neurons and the related synchrony of cortical neurons. ${ }^{11}$ Indeed, stimulation of brainstem cholinergic nuclei has been shown to result in widespread cortical gamma oscillations. ${ }^{6}$ The reticular formation can thus modulate the state of the cerebral cortex via projections to the intralaminar and reticular nuclei of the thalamus, as well as through projections to the basal forebrain nuclei and the cortex directly. ${ }^{12}$ Four families of brainstem nuclei that influence the cerebral cortex are considered to play a role in consciousness, i.e. the classical reticular nuclei, the monoaminergic nuclei, the cholinergic nuclei and the autonomic nuclei. The classical reticular nuclei are situated in the brainstem core and project to the basal nuclei, as well as to the intralaminar thalamic nuclei. ${ }^{12}$ The monoaminergic nuclei include noradrenergic, dopaminergic and serotonergic nuclei. These nuclei project directly to the cortex, in order to modulate wakefulness and mood, as well as to the basal nuclei. ${ }^{12}$ Noradrenergic and serotonergic nuclei have been associated with attentiveness and behavioural responses to stimuli, while dopamine is, among others, associated with working memory by modulating excitatory and inhibitory transmission in the prefrontal cortex ${ }^{13}$, and with motor control and reward. ${ }^{12}$ It is, however, important to note that noradrenergic neurons are silent during both REM sleep and cataplexy attacks, in which awareness is retained. ${ }^{1}$ Cataplexy attacks are characterized by muscular weakness, slurred speech and impaired vision, however, hearing and awareness remain normal. ${ }^{14}$ It has therefore been suggested that noradrenergic neurons are not an absolute requirement for consciousness. ${ }^{1}$

Serotonergic neurons are similarly silenced during REM sleep ${ }^{15}$, but increase during deep sleep. ${ }^{16}$ They are therefore believed to be associated with the formation of neural assemblies that are not sufficient to generate consciousness. ${ }^{16}$ Central nervous system cholinergic nuclei ${ }^{12}$ include two major pathways: from the brainstem to the thalamus and from the brainstem via the basal nuclei to the cortex, thalamus, hippocampus and amygdala. ${ }^{1}$ Spiking activity in cholinergic neurons increases during wakefulness and REM sleep and decreases during non-REM or deep sleep. ${ }^{1}$ Acetylcholine is therefore believed to elevate arousal in the brain so as to allow the formation of consciousness, however fleeting. ${ }^{16}$ The brain stem autonomic nuclei are responsible for the control of visceral functions and project to the intralaminar thalamic nuclei, the basal forebrain and other brainstem nuclei. ${ }^{12}$ They could possibly be seen as consciousness enablers - a concept discussed later in this writing

Studies have shown that lesions of the mesencephalic reticular formation result in loss of consciousness. ${ }^{17}$ Indeed, lesions in the central and dorsal regions of the reticular formation, from midpons to midbrain often result in coma, vegetative states, or worse. ${ }^{12}$ According to Seth, it is thus the brainstem that maintains consciousness and the cerebral cortex and thalamus that in turn determine the contents of that consciousness. ${ }^{3}$ The neural correlates of awareness therefore include the brainstem, which maintains arousal via modulatory neurotransmission, as well as the cortex and thalamus, which determine the contents of the awareness. The fact that the cortex is essential for awareness is obvious since the brainstem and intralaminar thalamic nuclei receive little sensory input from the cortex and thus exhibit no explicit representation of the sensory attributes of stimuli. ${ }^{1}$ Therefore, they cannot support the contents of stimulus awareness, and are described as enablers, not contentproviders.

\section{Prefrontal cortex and consciousness}

The frontoparietal network plays a central role in the consciousness process. It is associated with higher cognitive functions such as planning, decision-making, problemsolving, distinguishing between appropriate and inappropriate actions, abstract thinking, rule learning, processing of spatial information, response selection, longterm memory retrieval, introspection, the way we experience emotions, the way we join perceptions into a unifying whole and the way we confer meaning to our perceptions. It is also essential in the process of focusing attention by detecting conflict between inputs and amplifying prefrontal activity which is biased towards a certain neural pathway, as well as by keeping internally generated stimuli in focus. In fact, it is often referred to as the executive brain and has even been associated with the enigma referred to as free will. 2,13,18,19,20 However, what the prefrontal cortex is perhaps best known for is its essential role in working memory, i.e. to keep relevant information in mind at a specific moment of planning and perceiving. Despite the fact that different cognitive functions are strongly dependent on specific areas of the prefrontal cortex, it would appear that these functions are accomplished by interconnected circuits of the prefrontal cortex as a whole. ${ }^{13}$ Although there can be no doubt that the prefrontal cortices are essential for a high quality consciousness, it is important to remember their dependence on proper functioning of, and inflow from, the systems involved in arousal, the limbic system and other cortical areas. In other words these 
unconscious processes are essential for consciousness. They do not, however, represent consciousness. The essential role of these unconscious processes in the generation of consciousness is discussed by Gazzaniga in "The Mind's Past". ${ }^{21}$

The burning question therefore remains whether consciousness can exist without the contribution of the prefrontal cortex. It is assumed that passive awareness in contrast to active employment of the prefrontal area's functions can exist. ${ }^{22}$ Such awareness or consciousness in animals is described by LeDoux ${ }^{13}$ as "passive awareness as opposed to active use of on-line information", as occurs when the prefrontal cortices are involved. This passive awareness or off-line processing, as well as functions such as sensory consciousness, domain-specific consciousness and domainindependent consciousness are generally ascribed to animals that do not have the prefrontal capabilities of mankind. ${ }^{13}$ Who is to say that such types of consciousness do not occur in humans - both transiently on their own and continuously but masked by superimposed prefrontal higher order consciousness?

\section{Consciousness involves widespread brain activity}

It is obvious that, even at the lowest level of consciousness, the different areas considered essential for consciousness cannot function in isolation. It is therefore reasonable to accept that consciousness involves widespread brain activity. This, however, does not necessarily imply homogenous activity throughout the brain. During consciousness, brain activity is distributed throughout diffuse regions of the brain, while in unconscious states activity is said to remain localized. ${ }^{3}$ It is this widespread activity that has been said to mediate the unity of consciousness. It has further been advocated that mental health depends on the growth of neural circuits which ensure the integration of widely distributed brain areas into a functional whole. ${ }^{23}$ Trauma can impair this integration, with abused and neglected individuals displaying negative effects in the integrative corpus callosum and hippocampus. ${ }^{23}$ Although it is generally accepted that the different features of objects are processed in different areas of the brain, and that the different qualities are cognitively bound together and experienced against the background of previous experiences, the question of how this binding is accomplished remains. The cognitive binding problem thus attempts to address the mechanism/s by which we, as humans, experience our reality as seamless. Another facet of the binding problem is how the information from multiple objects is kept distinct. ${ }^{24}$ Currently, there are three hypothesized solutions to the binding problem, but the possibility exists that the final binding product is due to an interaction between all three modes of binding. ${ }^{25}$ The three major theories which attempt to explain binding are binding by convergence, binding by assembly and binding by synchrony. The first, i.e. binding by convergence, advocates that information from lower-order neurons is bound by higherorder neurons. ${ }^{25}$ This set of higher-order neurons then fires as an integrated binding unit once the full set of inputs have been received. ${ }^{25}$ Criticism of this theory involves the question of whether unambiguous binding can truly exist. ${ }^{25} \mathrm{~A}$ binding unit for every distinct input would be required, which would then limit the flexibility of the connections. ${ }^{25}$ Furthermore, the question of how novel objects would be bound arises. This would require a population of uncommitted neurons with flexibility in their connections. ${ }^{25}$ The second, i.e. binding by assembly, promotes the idea that a binding unit consists of a Hebbian cell assembly that interconnects a distinct set of neurons. ${ }^{26}$ In a Hebbian cell assembly increases in synaptic efficiency within a circuit of neurons occurs due to repeated and persistent presynaptic stimulation of the postsynaptic cells. ${ }^{14}$ This persistent stimulation causes growth or metabolic changes in the cells such that the synaptic connections between them are strengthened. ${ }^{14}$ According to the binding by assembly theory the formation of complex Hebbian assemblies, which consist mainly of NMDA synapses, underlies consciousness. ${ }^{17}$ This approach allows for more flexibility than binding by convergence, since each neuron can participate in more than one assembly. Multiple binding patterns may therefore exist. ${ }^{25}$ Criticism, however, includes the problem of superposition, whereby a neuron will not be able to function adequately if both objects it may represent are presented simultaneously. ${ }^{25}$ The third possibility, i.e. binding by synchrony, supports the idea that the correlation between neurons occurs in the temporal dimension. As previously mentioned, it is believed that distinct neural subpopulations of the cortex are synchronized due to $40 \mathrm{~Hz}$ oscillations of the thalamus. ${ }^{27}$ Binding therefore occurs due to temporal coherence of thalamocortical activation of pyramidal cells, with the activation returning to the thalamus in a resonant thalamocortical re-activation loop. ${ }^{5}$ The synchronized discharge is believed to increase the postsynaptic impact of the neurons without altering their firing rate. ${ }^{24}$ This approach gives rise to more flexibility than both binding by convergence and assembly and is believed to entail a virtually endless coding capacity. ${ }^{6}$ An alteration in these 40 $\mathrm{Hz}$ oscillations would instinctively result in a change in the level of consciousness. Anaesthesia and Lysergic Acid Diethylamide (LSD), which both result in altered states of consciousness, are believed to cause the uncoupling of these $40 \mathrm{~Hz}$ cortical oscillations. ${ }^{10}$ However, this theory of unbinding has also been criticized. ${ }^{10}$ Koch advocates that the synchronization simply helps one coalition of neurons dominate over competing coalitions, especially during formation, and that $40 \mathrm{~Hz}$ oscillations are therefore not essential for consciousness. ${ }^{28}$

Another theory which advocates the involvement of a temporal dimension in consciousness, is the idea of a neural time factor. ${ }^{29}$ This hypothesis states that a minimum uninterrupted processing time of 200-500 milliseconds is required in order to elicit a conscious experience. ${ }^{2,29}$ An important conclusion that can be derived from this premise is the assumption that we therefore do not experience our sensory world immediately since each conscious experience is delayed. ${ }^{29}$ This delay would, however, be advantageous since it would provide the opportunity for modulatory influences to affect the content of the sensory experience. ${ }^{29}$ These modulatory influences may be stressrelated or voluntary and may push certain sensory information to the background so as to allow us to focus on other incoming information. ${ }^{30}$ Similarly, these influences may have the potential to completely block incoming information, such that an individual dissociates from their environment. ${ }^{30}$ 


\section{Memory and consciousness}

Working memory is by far the best studied and discussed type of memory with regard to consciousness. Various opinions exist as to the relationship between working memory and consciousness, including assumptions that working memory precedes, or rather can be present, without consciousness; that consciousness is the awareness of what is in working memory; that consciousness is a kind of working memory; that to be aware of something, (that) something must be in working memory; that the contents of working memory are what we are conscious of at a specific moment; and that although working memory expands the time frame of consciousness it is not necessarily essential for consciousness. ${ }^{13,31,32,33}$ Although the above-mentioned opinions, at first glance, appear to reflect fairly similar views on the relationship between working memory and consciousness, the subtle differences are of significance.

Perhaps one should first ask whether consciousness can exist without memory. According to Crick" 31 "some form of very short-term memory seems almost essential for consciousness, but this memory may be very transient, lasting for only a fraction of a second". What then is the relationship between short-term memory and consciousness? To be conscious of something at the time it occurs, that information must be represented in working memory at the time it occurs. In other words, working memory is the temporary storage depot of short-term memory. ${ }^{13}$ If one could extrapolate on the view of Nobel laureate Crick and his colleague Koch ${ }^{34,35}$ on conscious awareness of visual stimuli, it would appear that we may have conscious access to information processing when it occurs in areas with connections to the working memory circuitry of the prefrontal cortex, in other words, to the areas involved in working memory. However, working memory is not just about present occurrences and short-term memory. ${ }^{13}$ It underlies mental work that incorporates and manipulates previous experiences and knowledge stored in long-term memory stores with present perceptions and aims.

Looking at the assumption that working memory precedes consciousness we are reminded of the description in Kaplan and Sadock of consciousness operating at multiple levels of preconsciousness. ${ }^{36}$ Implicated, but not spelled-out, is the existence of an implicit working memory. Two other approaches to the relationship, i.e. that consciousness is the awareness of what is in working memory, and that the contents of working memory are what we are conscious of at a specific moment, stretch the involvement of consciousness too far as working memory is surely more than what we are consciously aware of. The next idea, that is, that consciousness is a kind of working memory, points towards multiple types of working memories - beyond explicit and perhaps implicit working memories. What seems to be a logical approach would be that to be aware of something, (that) something must be in working memory- keeping in mind that working memory is much more than what we are explicitly aware of. ${ }^{37}$ The question then remains whether the prefrontal cortex is an absolute necessity for consciousness, that is, whether consciousness can, even fleetingly, exist without the working memory function of the prefrontal cortex. Perhaps far-fetched, yet tempting, would be to suggest that domain-independent consciousness or even domain-specific consciousness, as in non-humans, still exists in man in frontal and perhaps also in other cortical areas, but that its presence is most of the time obscured by the highly developed prefrontal cortex-dependent consciousness. There are indeed indications that domain-specific regions do exist in the prefrontal cortex and that short-term memory may perhaps be dependent on these areas. ${ }^{13}$ It is, however, strongly suspected, and logical, that the executive functions, and by implication the higher order consciousness, is reliant on binding across the various areas of the prefrontal cortex. ${ }^{38}$ Perhaps the difference between the consciousness of mere basic awareness of self, without being aware of anything in particular, and extended or higher order consciousness lies in the degree of integration across the prefrontal areas. To use the popular phrase, it depends on what is "on-line".

The role of a very important function in the making of consciousness, emotion, has this far been ignored. Good descriptions of this function/system and its role in consciousness can be found in the prolific works of authors like Gazzaniga, LeDoux, Damasio, Schwartz and Begley, and others. If the level of consciousness at any specific moment depends on the extent of the "on-line" circuitry then the answer to what determines the latter would appear to be attention. The level of attention bestowed on something dictates to what extent the object or experience would be in conscious awareness. Attention therefore determines the level, direction and content of consciousness. ${ }^{39}$

\section{Conclusion}

In this discussion on the neural correlates of consciousness we have seen that consciousness is dependent on, at least, the brain stem and thalamus for arousal, that basic cognition is supported by recurrent electrical activity between the cortex and the thalamus at gamma band frequencies, and that some kind of working memory, at least fleetingly, must be present for awareness to occur. It is generally accepted that the different features of objects are processed in different areas of the brain, and that the different qualities are cognitively bound together and experienced against the background of previous experiences - a process known as cognitive binding. However, for consciousness to be endowed with meaning that binds our perceptions into the seamless unity suggested by Kant ${ }^{40}$ we require far-reaching explicit working memory which probably feeds on integrative circuits across the different domains of the prefrontal cortex. This functioning of the prefrontal areas can be modified by the neuromodulatory systems which have an influence on the degree of alertness and attention, affective state and the meaning of experiences. Furthermore, for the prefrontal structures to function properly in the generation of consciousness they must receive information from other brain areas such as other association cortices and the limbic system. Consciousness thus depends on the multitude of unconscious processes that occurs in the brain. Although they don't represent consciousness, consciousness cannot exist without these processes. The factor that determines which of the subconscious processes will reach consciousness, appears to be focussed attention. This act of deliberately bestowing attention on something specific draws attention to the enigma of free will. 


\section{References}

1. Koch C. The quest for consciousness: A neurobiological approach. Colorado: Roberts and Company Publishers, 2004:87-104.

2. Hudetz AG. Suppressing consciousness: Mechanisms of general anesthesia. Seminars in Anesthesia, Perioperative Medicine and Pain 2006;25:196-204.

3. Seth AK, Baars BJ, Edelman DB. Criteria for consciousness in humans and other mammals. Conscious Cogn 2005;14;119-39.

4. Ratey JJ. A user's guide to the brain: Perception, attention and the four theatres of the brain. New York: Vintage Books, 2002:1 10-46.

5. Sadock BJ, Sadock VA (editors). Kaplan and Sadock's comprehensive textbook of psychiatry. 8th edition. Philadelphia: Lippincott Williams \& Wilkins, 2005:575-82

6. Buzsáki G. Rhythms of the Brain. New York: Oxford University Press, 2006:231-61

7. Zeman A. Consciousness. Brain 2001;124:1263-89.

8. Llinás $R$, Paré D. Of dreaming and wakefulness. Neuroscience 1991;44:119-39.

9. Massimini M, Ferrarelli F, Huber R, Esser SK, Singh H, Tononi G. Breakdown of cortical effective connectivity during sleep. Science 2005;309:2228-32.

10. The sinking brain: How to measure consciousness in anaesthesia. Best Pract Res Clin Anaesthesiol 2006;20(1):1-9

11. Purves D, Augustine GJ, Fitzpatrickj D, Hall WC, LaMantia A, McNamara JO, editors. Neuroscience. 3rd edition. Massachusetts: Sinauer Associates Inc,2004:679-81.

12. Parvizi J, Damasio A. Consciousness and the brainstem. Cognition 2001;79(1-2):135-60

13. LeDoux J. Synaptic self: how our brains become who we are. New York: Viking,2002:175-99.

14. Wikipedia [database on the Intenet]. c2001- [cited 2008 Jan 23]. Available from: http://www.wikipedia.org.

15. Damasio A. The feeling of what happens: Body and emotion in the making of consciousness. San Diego: Harcourt Inc, 1999:234-76.

16. Greenfield SA. Journey to the centres of the mind: toward a science of consciousness. New York: W.H. Freeman and Company, 1995:137-62.

17. Flohr H. An information processing theory of anaesthesia. Neuropsychologia 1995;33(9):1169-80.

18. Miller EK, Cohen JD. An integrative theory of prefrontal cortex function. Annu Rev Neurosci 2001;24:167-202

19. Squire LR, Bloom FE, MCConnell SK, Roberts JL, Spitzer NC, Zigmond $M J$, editors. Fundamental Neuroscience. 2nd edition. Academic Press, 2003:1353-94.

20. Carter R. Mapping the mind. California: University of California
Press, 1999: 180-207.

21. Gazzaniga MS. The Mind's Past. Berkeley: University of California Press, 1998.

22. Panksep J. Affective Neuroscience. New York: Oxford University Press, 1998.

23. Sadock BJ, Sadock VA (editors). Kaplan and Sadock's comprehensive textbook of psychiatry. 8th edition. Philadelphia: Lippincott Williams \& Wilkins, 2005:516-20.

24. Koch C. The quest for consciousness. A neurobiological approach. Colorado: Roberts and Company Publishers, 2004:303-14.

25. Mashour GA. Consciousness Unbound. Toward a paradigm of general anesthesia. Anesthesiology 2004;100:428-33.

26. van Essen DC, Anderson CH, Felleman DJ. Information processing in the primate visual system: An integrated systems perspective. Science 1992;255:419-23.

27. Mashour GA. Integrating the science of consciousness and anesthesia. Anesth Analg 2006;103(4):975-82.

28. Koch C. The quest for consciousness: A neurobiological approach. Colorado: Roberts and Company Publishers, 2004:315-27.

29. Libet B. How does conscious experience arise? The neural time factor. Brain Res Bull 1999;50(5/6):339-40.

30. Viljoen M. Stress and PNI [CD-ROM]. Pretoria: Department of Telematic Learning and Education Innovation;2004.

31. Crick F. Consciousness - not a thing but a process. In: Carter R. Mapping the mind. California: University of California Press, 1999:204-5.

32. Kosslyn SM, Koenig O. Wet mind: the new cognitive neuroscience. New York: MacMillam, 1992.

33. Johnson-Laird PN. The computer and the mind: an introduction to cognitive science. Cambridge: Harvard University Press, 1988.

34. Crick FC, Koch C. Some reflections on visual awareness. Cold Spring Harb Symp Quant Biol 1990;55:953-62.

35. Crick FC, Koch C. Are we aware of neural activity in primary visual cortex? Nature 1995;375:121-3.

36. Sadock BJ, Sadock VA (editors). Kaplan and Sadock's comprehensive textbook of psychiatry. 8th edition. Philadelphia: Lippincott Williams \& Wilkins, 2005:980-3.

37. LeDoux J. The emotional brain: the mysterious underpinnings of emotional life. New York: Touchstone, 1996:267-303.

38. Smith EE, Jonides J. Storage and executive processes in the frontal lobes. Science 1999;283(5408):1657-61.

39. Damasio A. The feeling of what happens: Body and emotion in the making of consciousness. San Diego: Harcourt Inc, 1999:1-31.

40. Kant I. Critique of pure reason. New York: St. Martin's Press, 1965. 